\title{
Hirota's method and the search for integrable partial difference equations. 1. Equations on a $3 \times 3$ stencil
}

\author{
Jarmo Hietarinta ${ }^{1 *}$ and Da-jun Zhang $^{2 \dagger}$ \\ ${ }^{1}$ Department of Physics and Astronomy, University of Turku, FIN-20014 Turku, Finland \\ ${ }^{2}$ Department of Mathematics, Shanghai University, Shanghai 200444, P.R. China
}

September 20, 2018

\begin{abstract}
Hirota's bilinear method ("direct method") has been very effective in constructing soliton solutions to many integrable equations. The construction of one- and two-soliton solutions is possible even for non-integrable bilinear equations, but the existence of a generic three-soliton solution imposes severe constraints and is in fact equivalent to integrability. This property has been used before in searching for integrable partial differential equations (PDE), and in this paper we apply it to two dimensional partial difference equations $(\mathrm{P} \Delta \mathrm{E})$ defined on a $3 \times 3$ stencil. We also discuss how the obtained equations are related to projections and limits of the three-dimensional master equations of Hirota and Miwa, and find that sometimes a singular limit is needed.
\end{abstract}

\section{Introduction}

Integrable dynamics is fundamentally associated with regularity and predictability, but if the equation is nonlinear this does not imply that the behavior is simple, quite the contrary.

There are in fact many interesting mathematical properties that are associated with nonlinear integrable PDE's (soliton equations), such as the existence of sufficient number of conserved quantities and symmetries, the ability to write the PDE as the consistency condition of two linear equations (the Lax pair, with spectral parameter), the existence of multi-soliton solutions, etc.[1]. Any of these characterizing properties can also be used

\footnotetext{
*E-mail: jarmo.hietarinta@utu.fi

†E-mail: djzhang@staff.shu.edu.cn
} 
to search for integrable PDE's and it is remarkable that in the end each criterion gives essentially the same set of equations.

In recent years the study of integrable partial difference equations $(\mathrm{P} \Delta \mathrm{E})$ has progressed rapidly [2]. The above integrability indicators have also been applied to P $\Delta \mathrm{E}$ 's, but in the discrete case there are also other criteria, specific to discrete equations, such as singularity confinement[3], the so called "Consistency-Around-the-Cube" property [4], and slow growth of complexity of the iterates (algebraic entropy)[5].

In this paper we consider the existence of three-soliton solutions for $\mathrm{P} \Delta \mathrm{E}$ 's as an indicator of integrability. We use Hirota's bilinear method, which is well suited for constructing soliton solutions. In Section 2 we first describe how the method works in searching for integrable PDE's and then formulate the search problem for P $\Delta$ E's. In Section 3 we give the results for a particular class of two-dimensional lattice equations, namely those defined on a $3 \times 3$ stencil. It is remarkable that all of the integrable equations obtained from this class can in fact be described as projections and limits of the well known threedimensional bilinear master-equations or Hirota [6] or Miwa [7], although sometimes a singular limit is needed. This is shown in Section 4.

\section{Hirota's direct method}

\subsection{The continuum case}

In 1971 R. Hirota proposed a direct method for constructing soliton solutions [8]. A key ingredient in this method is a transformation to new dependent variables so that in these new variables a soliton solution is a finite sum of exponentials.

\subsubsection{Definition}

For the prototypical example of KdV equation

$$
u_{t}+u_{x x x}+6 u u_{x}=0
$$

one applies the transformation

$$
u=2 \partial_{x}^{2} \log F
$$

and after integrating the resulting equation once one gets a bilinear equation

$$
\left(D_{x}^{4}+D_{x} D_{t}\right) F \cdot F=0,
$$

were the Hirota derivative operator $D$ is defined by

$$
\begin{aligned}
D_{x}^{n} f \cdot g & =\left.\left(\partial_{x_{1}}-\partial_{x_{2}}\right)^{n} f\left(x_{1}\right) g\left(x_{2}\right)\right|_{x_{2}=x_{1}=x} \\
& \left.\equiv \partial_{y}^{n} f(x+y) g(x-y)\right|_{y=0} .
\end{aligned}
$$

Thus $D$ acts on a product similarly to the Leibniz rule, except for a crucial change of sign. In particular $D_{x}^{n} f \cdot g=(-1)^{n} D_{x}^{n} g \cdot f$. Equations in which derivatives appear only through 
the above $D$-operator are said to be in Hirota bilinear form. (For further properties of the $D$ operator, see the book [9] and Appendix I of [10].)

The first step in constructing solitons solutions is therefore to find the dependent variable transformation that leads to a Hirota bilinear form. Unfortunately the bilinearization of a given nonlinear PDE is not easy. One cannot know before hand how many dependent or even independent variables one need to properly bilinearize a given equation. ${ }^{1}$

\subsubsection{Gauge invariance}

At this point we would like to emphasize that equations written in Hirota bilinear form can also be characterized by gauge invariance. Consider the gauge transformation $(f, g, \ldots) \mapsto$ $\left(e^{\vec{x} \cdot \vec{p}} f, e^{\vec{x} \cdot \vec{p}} g, \ldots\right)$, then the condition for gauge invariance is

$$
P(\vec{D})\left(e^{\vec{x} \cdot \vec{p}} f\right) \cdot\left(e^{\vec{x} \cdot \vec{p}} g\right)=e^{2 \vec{x} \cdot \vec{p}} P(\vec{D}) f \cdot g .
$$

(Here we use vector notation: $\vec{D}=\left(D_{x}, D_{y}, D_{z}, \ldots\right), \vec{x}=(x, y, z, \cdots), \vec{p}=\left(p_{x}, p_{y}, p_{z}, \cdots\right)$ with inner product $\vec{x} \cdot \vec{p}=p_{x} x+p_{y} y+\cdots$.) Equations written in Hirota bilinear form have this property, and conversely, any quadratic equation having gauge invariance of the above type can be written in Hirota bilinear form [11].

Gauge invariance allows generalizations of the Hirota bilinear form to other circumstances, such as higher multi-linearity [11] and discrete equations.

\subsubsection{SS and 2SS}

The existence of a bilinear form does not guarantee the existence of $N$-soliton solutions, but one- and two-soliton solutions are often easy to construct. As an example take the bilinear equation

$$
P(\vec{D}) F \cdot F=0
$$

where $P$ is some even polynomial in $\vec{D}$ (such as (1) in the KdV case).

In order to start the expansion we need a vacuum or background solution, which in this case is $F \equiv 1$ (which in the $\mathrm{KdV}$ case corresponds to $u \equiv 0$ ). This works, provided that $P(0)=0$.

Starting with the vacuum $F=1$ one builds a one-soliton solution (1SS) "perturbatively" using exponentials of linear functions, which are called plane wave factors (PWF):

$$
F=1+\epsilon e^{\eta_{i}} \text {, where } \eta_{i}=\vec{x} \cdot \vec{p}_{i}+\eta_{i}^{0} \text {, with constant } \eta_{i}^{0} .
$$

Here $\epsilon$ is the formal expansion parameter, and upon substituting this to (4) one finds the constraint that the parameters $\vec{p}_{i}$ must satisfy the dispersion relation (DR)

$$
P\left(\vec{p}_{i}\right)=0
$$

\footnotetext{
${ }^{1}$ In the literature one sometimes finds examples of over-bilinearization, in which a multi-linear equation is broken down into too many bilinear equations. One case study can be found in [12].
} 
Continuing in this manner one finds that the generic equation (4) also has two-soliton solutions of the form

$$
F=1+\epsilon e^{\eta_{1}}+\epsilon e^{\eta_{2}}+\epsilon^{2} A_{12} e^{\eta_{1}+\eta_{2}}
$$

where

$$
A_{i j}=-\frac{P\left(\vec{p}_{i}-\vec{p}_{j}\right)}{P\left(\vec{p}_{i}+\vec{p}_{j}\right)} .
$$

Here each $\vec{p}_{i}$ is restricted by the DR (6), that already appeared in constructing the 1SS, but there are no additional restrictions on them. $A_{i j}$ is called the phase factor. Note that $A_{i j}=A_{j i}$ due to $P$ being even.

In the above constructions crucial role is played by the minus sign in the definition (2) of the Hirota derivative, which implies

$$
P(\vec{D}) e^{\vec{x} \cdot \vec{p}} \cdot e^{\vec{x} \cdot \vec{p}^{\prime}}=P\left(\vec{p}-\vec{p}^{\prime}\right) e^{\vec{x} \cdot\left(\vec{p}+\vec{p}^{\prime}\right)}
$$

In practice this means that the first and the last terms of expressions like the 1SS (5) and 2SS (7) automatically satisfy the equation (if $P(0)=0$ ).

The above construction shows a level of partial integrability: we can have elastic scattering of two solitons, for any dispersion relation, if the nonlinearity is suitable (namely if it arises from a bilinear equation as above).

\subsubsection{The 3SS-condition}

Definition 1 (Hirota integrability)

Assume that the bilinear equation (4) has $1 S S$ 's of the form

$$
F=1+\epsilon e^{\eta_{j}}, \quad \eta_{j}=\vec{x} \cdot \vec{p}_{j}+\eta_{j}^{0},
$$

where the parameters $\vec{p}_{j}$ are only restricted by the DR $P\left(\vec{p}_{j}\right)=0$. The equation is said to be Hirota integrable if it has $N S S$ 's of the form

$$
F=1+\epsilon \sum_{j=1}^{N} e^{\eta_{j}}+(\text { finite number of h.o. terms in } \epsilon)
$$

without any further conditions on the parameters $\vec{p}_{j}$ of the individual solitons.

The assumption that there are no further restrictions is important, because any equation has multi-soliton solutions for some restricted set of parameters.

If we now apply this principle to the 3SS we start with the ansatz

$$
\begin{aligned}
F= & 1+\epsilon e^{\eta_{1}}+\epsilon e^{\eta_{2}}+\epsilon e^{\eta_{3}} \\
& +\epsilon^{2} A_{12} e^{\eta_{1}+\eta_{2}}+\epsilon^{2} A_{23} e^{\eta_{2}+\eta_{3}}+\epsilon^{2} A_{31} e^{\eta_{3}+\eta_{1}}+\epsilon^{3} A_{12} A_{23} A_{13} e^{\eta_{1}+\eta_{2}+\eta_{3}},
\end{aligned}
$$

which is fixed by the requirement that if in a NSS any soliton goes far away, the rest should look like the (N-1)SS. (In practice "going away" means either $e^{\eta_{k}} \rightarrow 0$ or $e^{\eta_{k}} \rightarrow \infty$.) This 
means that there is no freedom left: parameters are restricted only by the dispersion relation (6) and the phase factors $A_{i j}$ were given already in (8). Thus the existence of three-soliton solutions is not automatic, but rather it imposes severe requirements on the polynomial $P$.

The above definition of integrability can be used as a method for searching for new integrable equations. In practice it means that one should find polynomials $P$ that verify the "three-soliton-condition" (3SC), which follows when the ansatz (10) is substituted into (4), namely

$$
\begin{aligned}
\sum_{\sigma_{i}= \pm 1} P\left(\sigma_{1} \vec{p}_{1}+\right. & \left.\sigma_{2} \vec{p}_{2}+\sigma_{3} \vec{p}_{3}\right) P\left(\sigma_{1} \vec{p}_{1}-\sigma_{2} \vec{p}_{2}\right) \\
& \times P\left(\sigma_{2} \vec{p}_{2}-\sigma_{3} \vec{p}_{3}\right) P\left(\sigma_{1} \vec{p}_{1}-\sigma_{3} \vec{p}_{3}\right)=0,
\end{aligned}
$$

on the manifold $P\left(\vec{p}_{i}\right)=0, \forall i$. If the $3 \mathrm{SC}$ is satisfied then the equation most likely has NSS of the form

$$
F=\sum_{\mu_{i} \in\{0,1\}} \exp \left[\sum_{i>j}^{(N)} a_{i j} \mu_{i} \mu_{j}+\sum_{i=1}^{N} \mu_{i} \eta_{i}\right], \quad \text { where } \quad \exp \left(a_{i j}\right)=A_{i j} .
$$

\subsubsection{Continuous searches}

Condition (11) can be easily converted for symbolic computation, for example in REDUCE [13] one can use the LET-rule for the dispersion relations (which in REDUCE is evaluated to the end). In 1987-1988 one of the present authors (JH) used this method to search for

new integrable equations of $\mathrm{KdV}, \mathrm{mKdV}$, sG and nlS type [14, 15], for which conditions similar to (11) can be derived. Among the (very few) new equations found in this way were

$$
\begin{array}{r}
\left(D_{x}^{3} D_{t}+a D_{x}^{2}+D_{t} D_{y}\right) F \cdot F=0, \\
\left(D_{x}^{4}-D_{x} D_{t}^{3}+a D_{x}^{2}+b D_{x} D_{t}+c D_{t}^{2}\right) F \cdot F=0,
\end{array}
$$

and

$$
\left\{\begin{aligned}
\left(i \alpha D_{x}^{3}+3 D_{x} D_{y}-2 i D_{t}+c\right) G \cdot F & =0 \\
{\left[a\left(\alpha^{2} D_{x}^{4}-3 D_{y}^{2}+4 \alpha D_{x} D_{t}\right)+b D_{x}^{2}\right] F \cdot F } & =|G|^{2} .
\end{aligned}\right.
$$

The pair of equations (15) is of the nonlinear Shrödinger type and in fact combines the two most important $(2+1)$-dimensional equations, Davey-Stewartson and KadomtsevPetviashvili equations, but only their DSII and KPI variants.

\subsection{Hirota's bilinear formalism for lattice equations}

We now turn to discrete lattice equations and as a model we use the continuous situation described above. The various differences between continuous and discrete models (such as the lack of Leibniz rule in the latter) make things now much more difficult. We will only consider $\mathrm{P} \Delta \mathrm{E}$ 's defined on the 2-dimensional cartesian lattice. 


\subsubsection{Discrete Hirota bilinear form}

Before we can proceed to equations we must have a definition of the discrete Hirota bilinear form. The guiding property is still the gauge-invariance (3), and thus assuming we have lattice functions $f_{j}(n, m)$, we require gauge-invariance under

$$
f_{j}(n, m) \mapsto f_{j}^{\prime}(n, m)=A^{n} B^{m} f_{j}(n, m) .
$$

Guided by the requirement of invariance under (16) we have

Definition 2 (Discrete Hirota bilinear form) If the equation can be written as

$$
\sum_{j} c_{j} f_{j}\left(n+\nu_{j}^{+}, m+\mu_{j}^{+}\right) g_{j}\left(n+\nu_{j}^{-}, m+\mu_{j}^{-}\right)=0
$$

where the index sums $\mu_{j}^{+}+\mu_{j}^{-}=\mu^{s}, \nu_{j}^{+}+\nu_{j}^{-}=\nu^{s}$ do not depend on $j$, we say it has discrete Hirota bilinear form.

Note that

$$
e^{a D_{x}} f(x) \cdot g(x)=\left.e^{a\left(\partial_{x}-\partial_{x^{\prime}}\right)} f(x) g\left(x^{\prime}\right)\right|_{x^{\prime}=x}=f(x+a) g(x-a) .
$$

Therefore the discrete version of $P(D) f \cdot f=0$ should be an even function built up from exponentials of the Hirota derivative. For example a sum containing terms like $\cosh \left(D_{x}\right)$ or $\sinh \left(D_{x}\right) \sinh \left(D_{t}\right)$ etc., is acceptable.

\subsubsection{The Hirota and Miwa equations}

The first results on discrete bilinear soliton equations were obtained by Hirota in a series of papers in 1977 [16]. Hirota's aim was to discretize known soliton equations so that their multi-soliton solutions would still have more or less the same form. In this way he was able to derive integrable 2D lattice equations for KdV, Toda, and sine-Gordon equations. The culmination of this series was the "Discrete Analogue of a Generalized Toda Equation" (DAGTE) or "Hirota-equation" [6], which in the fully 3D version (discrete bilinear KP equation) can be written as [7]

$$
a(b-c) \tau_{n+1, m, k} \tau_{n, m+1, k+1}+b(c-a) \tau_{n, m+1, k} \tau_{n+1, m, k+1}+c(a-b) \tau_{n, m, k+1} \tau_{n+1, m+1, k}=0 .
$$

This equation has soliton solutions of the form (12), but with discretized PWF's given by $[7,17]$

$$
e^{\eta_{i}}=\left(\frac{1-a q_{i}}{1-a p_{i}}\right)^{n}\left(\frac{1-b q_{i}}{1-b p_{i}}\right)^{m}\left(\frac{1-c q_{i}}{1-c p_{i}}\right)^{k}, \quad A_{i j}=\frac{\left(p_{i}-p_{j}\right)\left(q_{i}-q_{j}\right)}{\left(p_{i}-q_{j}\right)\left(q_{i}-p_{j}\right)} .
$$

In the early 1980's the continuum bilinear approach was developed to a complete theory by Kyoto school, and it was very soon applied to the discrete case as well $[7,17]$. 
We note in particular the generalization of Hirota's equation (17a) by Miwa (the discrete BKP equation) [7]

$$
\begin{gathered}
(a+b)(a+c)(b-c) \tau_{n+1, m, k} \tau_{n, m+1, k+1}+(b+c)(b+a)(c-a) \tau_{n, m+1, k} \tau_{n+1, m, k+1} \\
+(c+a)(c+b)(a-b) \tau_{n, m, k+1} \tau_{n+1, m+1, k}+(a-b)(b-c)(c-a) \tau_{n+1, m+1, k+1} \tau_{n, m, k}=0 .
\end{gathered}
$$

Also this equation has soliton solutions of the form (12), except that now $[7,17]$

$$
\begin{aligned}
e^{\eta_{i}} & =\left(\frac{\left(1-a p_{i}\right)\left(1-a q_{i}\right)}{\left(1+a p_{i}\right)\left(1+a q_{i}\right)}\right)^{n}\left(\frac{\left(1-b p_{i}\right)\left(1-b q_{i}\right)}{\left(1+b p_{i}\right)\left(1+b q_{i}\right)}\right)^{m}\left(\frac{\left(1-c p_{i}\right)\left(1-c q_{i}\right)}{\left(1+c p_{i}\right)\left(1+c q_{i}\right)}\right)^{k}, \\
A_{i j} & =\frac{\left(p_{i}-p_{j}\right)\left(p_{i}-q_{j}\right)\left(q_{i}-p_{j}\right)\left(q_{i}-q_{j}\right)}{\left(p_{i}+p_{j}\right)\left(p_{i}+q_{j}\right)\left(q_{i}+p_{j}\right)\left(q_{i}+q_{j}\right)} .
\end{aligned}
$$

\subsubsection{Application of Hirota's method to some $\mathrm{P} \Delta \mathrm{E}$ 's}

Hirota's direct method has also been applied to construction of soliton solutions to nonlinear lattice equations. There the first problem is to construct the dependent variable transform that takes one from the nonlinear form to the Hirota bilinear form with gauge invariance. This approach was used for most of the equations in the ABS list in [18, 19]. For example, in the case of the $\mathrm{H} 1$ equation

$$
\mathrm{H} 1 \equiv(u-\widehat{\widetilde{u}})(\widetilde{u}-\widehat{u})+\left(a^{2}-b^{2}\right)=0,
$$

one is led (after studying the 1SS and 2SS) to the dependent variable transformation

$$
u_{n, m}^{(N S S)}=a n+b m+\lambda-\frac{g_{n, m}}{f_{n, m}}
$$

which yields the bilinearization

$$
\mathrm{H} 1=-\left[\mathcal{H}_{1}+(a-b) f \widehat{\widetilde{f}}\right]\left[\mathcal{H}_{2}+(a+b) \widehat{f} \widetilde{f}\right] /(f \widehat{f} \widetilde{f} \widehat{\widetilde{f}})+\left(a^{2}-b^{2}\right)
$$

where

$$
\begin{aligned}
& \mathcal{H}_{1} \equiv \widehat{g} \tilde{f}-\widetilde{g} \hat{f}+(a-b)(\widehat{f} \widetilde{f}-f \widehat{\widetilde{f}})=0, \\
& \mathcal{H}_{2} \equiv g \widehat{\widetilde{f}}-\widehat{\widetilde{g}} f+(a+b)(f \hat{\widetilde{f}}-\widehat{f} \widetilde{f})=0
\end{aligned}
$$

\section{Searching for integrable bilinear lattice equations}

Following the continuous example we would like to search for integrable lattice equations by the three-soliton condition. Since we will here only consider 1-component equations, we can still take (4) as the basic class of equations, but now $P$ would have to be a sum of exponentials. 


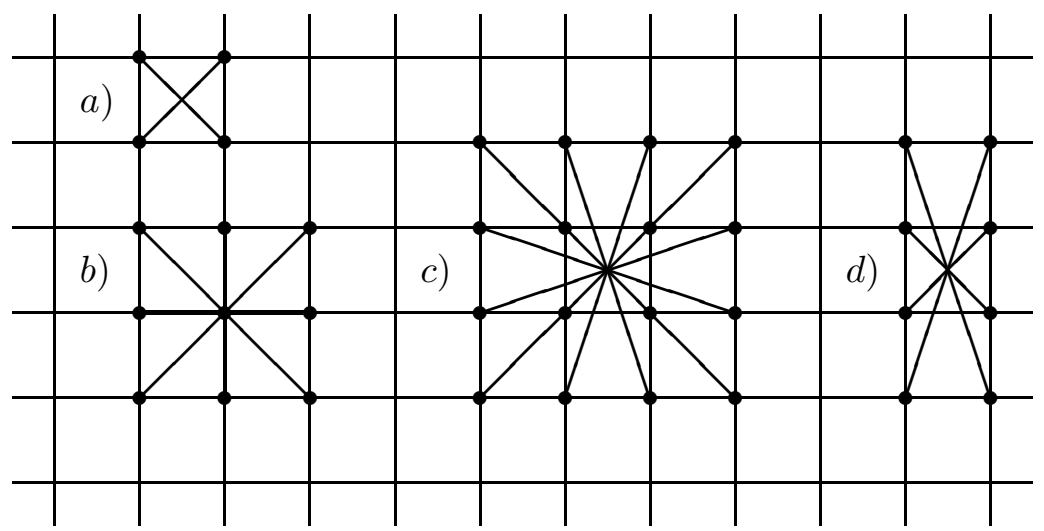

Figure 1: Possible stencils for 2D bilinear equations. Case a) only contains 1 equation (22), Case b) is analyzed in this paper, Case d) and similar models are analyzed in [20].

\subsection{Organizational questions}

The first question is: How to organize such a search? In the continuous case we used the total degree in the bilinear derivative and then the degrees in various coordinates. The problem in the discrete case is that there are so many ways to discretize a derivative and thus there will be many more cases.

One way to proceed is by the radius of points needed, see Figure 1. For sub-figure 1a) the corresponding bilinear equation will be

$$
c_{1} f_{n, m} f_{n+1, m+1}+c_{2} f_{n+1, m} f_{n, m+1}=0,
$$

since these are the only combinations of $f_{n+\nu, m+\mu} f_{n+\nu^{\prime}, m+\mu^{\prime}}$ with non-negative $\mu, \mu^{\prime}, \nu, \nu^{\prime}$ and sum $\mu+\mu^{\prime}=1, \nu+\nu^{\prime}=1$. Since we want $f \equiv 1$ to be a solution we must have $c_{1}+c_{2}=0$ and then there are no parameters, except the trivial scaling.

In this paper we will consider the arrangement given in sub-figure 1b) which corresponds to the difference equation

$$
\begin{aligned}
& g_{8} f_{n+1, m+1} f_{n-1, m-1}+g_{6} f_{n+1, m-1} f_{n-1, m+1} \\
& +g_{7} f_{n+1, m} f_{n-1, m}+h_{6} f_{n, m+1} f_{n, m-1}+h_{7} f_{n, m}^{2}=0 .
\end{aligned}
$$

In terms of Hirota's $D$-derivatives this can be written as (4), with the function

$$
P(X, Y):=g_{8} e^{X+Y}+g_{6} e^{X-Y}+g_{7} e^{X}+h_{6} e^{Y}+h_{7} .
$$

However, since the $D$-operator is antisymmetric and $P$ operates on a symmetric target $f \cdot f$, only the symmetric part of $P$ is relevant. Thus the proper form is

$$
P(X, Y):=g_{8} \cosh (X+Y)+g_{6} \cosh (X-Y)+g_{7} \cosh (X)+h_{6} \cosh (Y)+h_{7} .
$$

Since equation (9) still holds, the construction of 1SS and 2SS goes as usual, resulting with formulae (5-8), with $\eta_{j}=p_{j} n+q_{j} m+\eta_{j}^{0}$. In particular, from the 0SS $f_{n, m}=1, \forall n, m$ 
we get the condition

$$
g_{6}+g_{7}+g_{8}+h_{6}+h_{7}=0
$$

and from the $\operatorname{SSS} f_{n, m}=1+e^{\eta_{j}}$ we get the $\operatorname{DR} P\left(p_{j}, q_{j}\right)=0$, i.e.,

$$
g_{8} \cosh \left(p_{j}+q_{j}\right)+g_{6} \cosh \left(p_{j}-q_{j}\right)+g_{7} \cosh \left(p_{j}\right)+h_{6} \cosh \left(q_{j}\right)+h_{7}=0,
$$

while the formulae for $A_{i j}(8)$ and for the $3 \mathrm{SC}$ (11) hold as written.

The practical problem is the following: How to implement the dispersion relation (26) while keeping the phase factor and the 3SC manageable? It seems that in order to implement the DR we must rationalize it somehow, but then we will loose the additive form of the phase factor and the 3SC.

As the first step we replace

$$
e^{p_{j}}=p_{j}^{\prime}, e^{q_{j}}=q_{j}^{\prime}
$$

and introduce the function

$$
P^{\prime}(x, y):=(x y)^{-1}\left[g_{8}\left(x^{2} y^{2}+1\right)+g_{6}\left(x^{2}+y^{2}\right)+g_{7} y\left(x^{2}+1\right)+h_{6} x\left(y^{2}+1\right)+2 h_{7} x y\right],
$$

after which the dispersion relation is still $P^{\prime}\left(p_{j}^{\prime}, q_{j}^{\prime}\right)=0$, but

$$
A_{i j}=-\frac{P^{\prime}\left(p_{i}^{\prime} / p_{j}^{\prime}, q_{i}^{\prime} / q_{j}^{\prime}\right)}{P^{\prime}\left(p_{i}^{\prime} p_{j}^{\prime}, q_{i}^{\prime} q_{j}^{\prime}\right)}
$$

and correspondingly in the 3SC. This does not look bad, except that the DR is still hard to implement. Next we continue with

$$
p_{j}^{\prime}=\frac{P_{j}-1}{P_{j}+1}, \quad q_{j}^{\prime}=\frac{Q_{j}-1}{Q_{j}+1}
$$

so that

$$
f_{n, m}=1+c_{j}\left(\frac{P_{j}-1}{P_{j}+1}\right)^{n}\left(\frac{Q_{j}-1}{Q_{j}+1}\right)^{m} .
$$

Now the DR gets a nice form

$$
\mathcal{P}\left(P_{j}, Q_{j}\right):=\left(P_{j}^{2}-1\right)^{-1}\left(Q_{j}^{2}-1\right)^{-1}\left(o_{1} P_{j}^{2}+2 o_{3} P_{j} Q_{j}+o_{2} Q_{j}^{2}+o_{4}\right)=0
$$

where

$$
\begin{array}{ll}
o_{1}=g_{7}+h_{7}, & g_{6}=\frac{1}{4}\left(-o_{1}-o_{2}+2 o_{3}-o_{4}\right), \\
o_{2}=h_{6}+h_{7}, \quad \text { with inverse } & g_{7}=\frac{1}{2}\left(o_{1}-o_{2}+o_{4}\right), \\
o_{3}=g_{6}-g_{8}, & g_{4}\left(-o_{1}-o_{2}-2 o_{3}-o_{4}\right), \\
o_{4}=g_{7}+h_{6}, & h_{6}=\frac{1}{2}\left(-o_{1}+o_{2}+o_{4}\right), \\
h_{7}=\frac{1}{2}\left(o_{1}+o_{2}-o_{4}\right) .
\end{array}
$$

However, the simple additive property in $A_{i j}$ is lost, we find instead

$$
A_{i j}=-\frac{\mathcal{P}\left(\frac{1-P_{i} P_{j}}{P_{i}-P_{j}}, \frac{1-Q_{i} Q_{j}}{Q_{i}-Q_{j}}\right)}{\mathcal{P}\left(\frac{1+P_{i} P_{j}}{P_{i}+P_{j}}, \frac{1+Q_{i} Q_{j}}{Q_{i}+Q_{j}}\right)}
$$

and the $3 \mathrm{SC}$ is still much more complicated.

In the following we use the parametrization (28), which implies $(29,30)$. 


\subsection{Results}

The search was performed by computing the 3SS subject to the dispersion relation

$$
o_{1} P_{j}^{2}+2 o_{3} P_{j} Q_{j}+o_{2} Q_{j}^{2}+o_{4}=0 .
$$

The dispersion relation was implemented using LET-rule of REDUCE [13] on the leading monomial (in "gradlex" ordering). In REDUCE such rules are evaluated to the end.

- For the generic case we may assume that $o_{1} \neq 0$ and scale it to $o_{1}=1$, then we can use the rule: for all $n$ let $p p(n)^{\wedge} 2=-2 * o 3 * p p(n) * q q(n)-02 * q q(n)^{\wedge} 2-\circ 4$; The resulting $3 \mathrm{SC}$ is rather long but the coefficients of various powers of $Q_{i}$ and first powers of $P_{i}$ are relatively simple and one can proceed by solving them one by one.

- If $o_{1}=0$ then we may assume $o_{3} \neq 0$ and scale it to $o_{3}=-1 / 2$. Then we can solve for $P_{j}$ and use the substitution for all $\mathrm{n}$ let $\mathrm{pp}(\mathrm{n})=\left(\mathrm{o} 2 * \mathrm{qq}(\mathrm{n}){ }^{\wedge} 2+04\right) / \mathrm{qq}(\mathrm{n})$; Some of these cases are $n \leftrightarrow m$ reflections of $o_{1}=1, o_{2}=0$.

The process branches somewhat but when the results are collected and sub-cases eliminated we get the following cases:

1. $o_{1}=1, o_{2}=2 o_{3}-o_{4}-1$

$$
f_{n+1, m+1} f_{n-1, m-1} o_{3}+f_{n+1, m} f_{n-1, m}\left(o_{3}-o_{4}-1\right)+f_{n, m+1} f_{n, m-1}\left(-o_{3}+1\right)+f_{n, m}^{2}\left(-o_{3}+o_{4}\right)=0 .
$$

2. $o_{1}=1, o_{2}=-2 o_{3}-o_{4}-1$

$$
f_{n+1, m} f_{n-1, m}\left(o_{3}+o_{4}+1\right)+f_{n+1, m-1} f_{n-1, m+1} o_{3}-f_{n, m+1} f_{n, m-1}\left(o_{3}+1\right)-f_{n, m}^{2}\left(o_{3}+o_{4}\right)=0 .
$$

3. $o_{1}=1, o_{2}=1, o_{4}=0$

$$
f_{n+1, m+1} f_{n-1, m-1}\left(o_{3}+1\right)+f_{n+1, m-1} f_{n-1, m+1}\left(-o_{3}+1\right)-2 f_{n, m}^{2}=0 .
$$

4. $o_{1}=1, o_{2}=0, o_{4}=1$

$$
f_{n+1, m+1} f_{n-1, m-1}\left(o_{3}+1\right)-2 f_{n+1, m} f_{n-1, m}+f_{n+1, m-1} f_{n-1, m+1}\left(-o_{3}+1\right)=0 .
$$

5. $o_{1}=0, o_{3}=-1 / 2, o_{4}=o_{2}$

$$
f_{n+1, m+1} f_{n-1, m-1}\left(-2 o_{2}+1\right)+f_{n+1, m-1} f_{n-1, m+1}\left(-2 o_{2}-1\right)+4 f_{n, m+1} f_{n, m-1} o_{2}=0 .
$$

6. $o_{1}=0, o_{3}=-1 / 2, o_{4}=1-o_{2}$

$$
f_{n+1, m} f_{n-1, m}\left(-2 o_{2}+1\right)-f_{n+1, m-1} f_{n-1, m+1}+f_{n, m+1} f_{n, m-1}+f_{n, m}^{2}\left(2 o_{2}-1\right)=0 .
$$

7. $o_{1}=0, o_{3}=-1 / 2, o_{4}=-1-o_{2}$

$$
f_{n+1, m+1} f_{n-1, m-1}+f_{n+1, m} f_{n-1, m}\left(-2 o_{2}-1\right)-f_{n, m+1} f_{n, m-1}+f_{n, m}^{2}\left(2 o_{2}+1\right)=0 .
$$

For further analysis we restore the freedom in the scaled parameters and combine cases to the extent possible. The final results can then be divided into the following cases: 


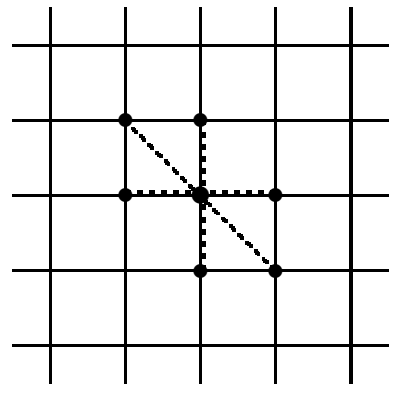

Case 1b)

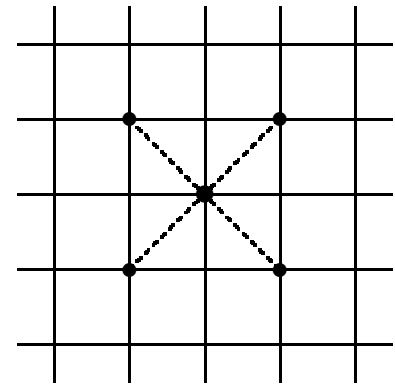

Case 2)

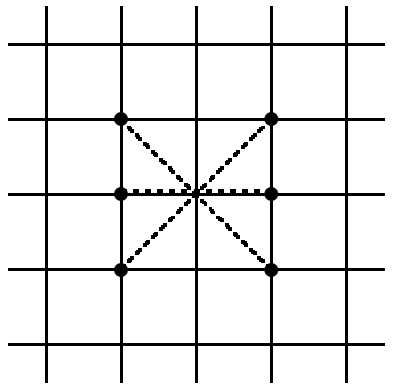

Case 3a)

Figure 2: Points involved in the equations with 3SS. Case $1 \mathrm{a}$ is obtained by a $90^{\circ}$ rotation from Case 1b, and Case 3b the same way from Case 3a.

Case 1: $o_{1}+o_{2}+o_{4}= \pm 2 o_{3}:$

$f_{n+1, m+1} f_{n-1, m-1} o_{3}+f_{n+1, m} f_{n-1, m}\left(o_{2}-o_{3}\right)+f_{n, m+1} f_{n, m-1}\left(o_{1}-o_{3}\right)+f_{n, m}^{2}\left(o_{3}-o_{1}-o_{2}\right)=0$.

$f_{n+1, m-1} f_{n-1, m+1} o_{3}-f_{n+1, m} f_{n-1, m}\left(o_{2}+o_{3}\right)-f_{n, m+1} f_{n, m-1}\left(o_{1}+o_{3}\right)+f_{n, m}^{2}\left(o_{3}+o_{1}+o_{2}\right)=0$.

These are related by $m \leftrightarrow-m$; due to (28) this corresponds to $Q \leftrightarrow-Q$, or in the dispersion relation, $o_{3} \leftrightarrow-O_{3}$. The associated diagram is given in Figure 2 .

For $o_{1}=1$ this contains items 1 and 2 of the previous list, and for $o_{1}=0, o_{3}=$ $-1 / 2, o_{4}=-1-o_{2}$ the first one also contains item 7 and for $o_{1}=0, o_{3}=-1 / 2, o_{4}=1-o_{2}$ the second one contains item 6 .

Case 2: $\quad o_{1}=o_{2} \neq 0, o_{4}=0$ (item 3 from the previous list):

$$
f_{n+1, m+1} f_{n-1, m-1}\left(o_{3}+o_{1}\right)+f_{n+1, m-1} f_{n-1, m+1}\left(-o_{3}+o_{1}\right)-2 o_{1} f_{n, m}^{2}=0 .
$$

This is the Toda-lattice (center + cross, see Figure 2).

Case 3: $o_{2}=0, o_{4}=o_{1}$ or $o_{1}=0, o_{4}=o_{2}\left(\right.$ or: $\left.o_{1}+o_{2}=o_{4}, o_{1}-o_{2}= \pm o_{4}\right)$.

$$
\begin{aligned}
& f_{n+1, m+1} f_{n-1, m-1}\left(o_{3}+o_{1}\right)+f_{n+1, m-1} f_{n-1, m+1}\left(o_{1}-o_{3}\right)-2 o_{1} f_{n+1, m} f_{n-1, m}=0 . \\
& f_{n+1, m+1} f_{n-1, m-1}\left(o_{2}+o_{3}\right)+f_{n+1, m-1} f_{n-1, m+1}\left(o_{2}-o_{3}\right)-2 o_{2} f_{n, m+1} f_{n, m-1}=0 .
\end{aligned}
$$

These contain item 4 and its $n \leftrightarrow m$ reflection, item 5 . Both are three term equations with two diagonals and a vertical or a horizontal, see Figure 2. They correspond to Hirota's discretization of the $\mathrm{KdV}$ equation [16].

\section{Post processing}

In this section we will take a closer look on the equations obtained, and in particular on how the soliton solutions look and how the final results can be obtained as a reduction 
from the Hirota or Miwa equations. In the following the parameters $o_{i}$ are from the search, $a, b, c$ are those used in the Hirota and Miwa equations (17a), (18a), and $\alpha, \beta$ are the canonical parameters in terms of which the PWF's have the simplest form.

\subsection{Case 2:}

\subsubsection{A better parameterization}

The dispersion relation for Case 2 reads

$$
o_{1} P^{2}+2 o_{3} P Q+o_{1} Q^{2}=0 .
$$

Parameterizing $o_{1}(\neq 0), o_{3}$ as

$$
o_{1}=2 \alpha \beta, \quad o_{3}=-\left(\alpha^{2}+\beta^{2}\right),
$$

the DR factorizes as

$$
(\alpha P-\beta Q)(\beta P-\alpha Q)=0 .
$$

Let us fix $\alpha, \beta$ as determined from (36). In the generic case $\alpha^{2} \neq \beta^{2}$ and we have in fact two different kinds of solitons, depending on which factor of (37) is satisfied. Thus we may choose either

$$
Q_{j}=\beta / k_{j}, \quad P_{j}=\alpha / k_{j},
$$

or

$$
Q_{j}=\alpha / k_{j}, \quad P_{j}=\beta / k_{j},
$$

and obtain the PWF

$$
e^{\eta_{i}}=\left(\frac{\alpha_{i}-k_{i}}{\alpha_{i}+k_{i}}\right)^{n}\left(\frac{\beta_{i}-k_{i}}{\beta_{i}+k_{i}}\right)^{m} \text {, where }\left(\alpha_{i}, \beta_{i}\right)=(\alpha, \beta) \text { or }(\beta, \alpha) .
$$

The phase factor $A_{i j}$ has the canonical form

$$
A_{i j}=\left(\frac{k_{i}-k_{j}}{k_{i}+k_{j}}\right)^{2} .
$$

\subsubsection{Reduction from Hirota's equation}

Equation (33) is obtained from (17a) by the reduction

$$
\tau(n+1, m+1, k)=\tau(n, m, k+1),
$$

which we use to define a new function $f$ by:

$$
\tau_{n, m, k}=f_{n+m+2 k, n-m} .
$$

Thus we define the new independent variables by

$$
n^{\prime}:=m+n+2 k-2, \quad m^{\prime}:=n-m
$$


and then it is easy to see that the 6 corner points of the cube on which (17a) is defined, namely $\{(100),(011),(010),(101),(001),(110)\}$ will project to $\{(-1,1),(1,-1),(-1,-1),(1,1)$, $(0,0),(0,0)\}$, displayed at the center of Figure 2 . With this definition one obtains $(33)$ for $f_{n^{\prime} m^{\prime}}$ with,

$$
o_{1}=\frac{1}{2} c(b-a), \quad o_{3}=\frac{1}{2}(c(a+b)-2 a b) .
$$

\subsubsection{Reduction of solution}

For the NSS of DAGTE given in (17b) the reduction (40) implies

$$
\left(\frac{1-a q_{i}}{1-a p_{i}}\right)\left(\frac{1-b q_{i}}{1-b p_{i}}\right)=\left(\frac{1-c q_{i}}{1-c p_{i}}\right)
$$

which has the nontrivial solution

$$
q_{i}=\frac{a b p_{i}-a-b+c}{a b\left(c p_{i}-1\right)} .
$$

Here we must assume that $a b(a-c)(b-c) \neq 0$. After this the PWF of (17b) gets the form

$$
e^{\eta_{i}}=\left(\sqrt{\frac{a-c}{b}} \sqrt{\frac{b-c}{a}} \frac{1}{c p_{i}-1}\right)^{n^{\prime}+2}\left(\sqrt{\frac{a-c}{b}} \sqrt{\frac{a}{b-c}} \frac{b p_{i}-1}{a p_{i}-1}\right)^{m^{\prime}} .
$$

We can write this in the canonical form for $2 \mathrm{D}$ systems

$$
e^{\eta_{i}}=\left(\frac{\alpha-k_{i}}{\alpha+k_{i}}\right)^{n}\left(\frac{\beta-k_{i}}{\beta+k_{i}}\right)^{m}
$$

where instead of $p$ we have used $k$ defined by

$$
p_{i}=\frac{1-u v}{c} \frac{k_{i}+s(u-v)(1+u v)}{k_{i}+s(u-v)(1-u v)}, \quad \text { where } \quad u:=\sqrt{\frac{a-c}{b}}, \quad v:=\sqrt{\frac{b-c}{a}},
$$

and then

$$
\alpha=s(u v-1)(u-v), \quad \beta=s(u v+1)(u+v) .
$$

Here $s$ is an arbitrary parameter. Note that for this case we have already derived PWF in the form (38), which allows different kinds of solitons by $\alpha \leftrightarrow \beta$. In the above the sign change in $u$ corresponds to this freedom.

\subsubsection{Special cases}

The case $o_{1}=o_{3}$ corresponds to $a(b-c)=0$ and $o_{1}=-o_{3}$ to $b(a-c)=0$, both of which break the formulae in Sec. 4.1.3. In fact in these cases the equation (33) only has two terms and the system can be reduced to the $1 \mathrm{D}$ equation $f_{N+1} f_{N-1}-f_{N}^{2}=0$.

The case $o_{3}=0$ corresponds to $\alpha= \pm i \beta$, which implies $c=2 a b /(a+b)$. The above formulae still hold for this special case. 


\subsection{Case 3a:}

\subsubsection{A better parameterization}

For Case 3 the DR is $\left(o_{1} o_{3} \neq 0\right)$

$$
o_{1} P_{i}^{2}+2 o_{3} P_{i} Q_{i}+o_{1}=0, \quad \text { i.e., } \quad Q_{i}=-\frac{o_{1}}{2 o_{3}}\left(P_{i}+1 / P_{i}\right),
$$

after which the PWF of (28) becomes

$$
e^{\eta_{i}}=\left(\frac{P_{i}-1}{P_{i}+1}\right)^{n}\left(\frac{o_{1} P_{i}^{2}+2 o_{3} P_{i}+o_{1}}{o_{1} P_{i}^{2}-2 o_{3} P_{i}+o_{1}}\right)^{m}, \quad A_{i j}=\left(\frac{\left(P_{i} P_{j}-1\right)\left(P_{i}-P_{j}\right)}{\left(P_{i} P_{j}+1\right)\left(P_{i}+P_{j}\right)}\right)^{2} .
$$

In order to simplify this we define a new soliton parameter $k_{i}$ by

$$
k_{i}:=\frac{2 \alpha P_{i}}{1+P_{i}^{2}},
$$

and furthermore let $o_{3}=-\alpha o_{1} / \beta$, after which we obtain

$$
e^{\eta_{i}}=\left(\frac{\alpha-k_{i}}{\alpha+k_{i}}\right)^{n / 2}\left(\frac{\beta-k_{i}}{\beta+k_{i}}\right)^{m}
$$

with $A_{i j}$ as in (39).

The appearance of $n / 2$ as the exponent in (45) means that the natural equation in this case is obtained by replacing $n=2 N$, i.e.,

$$
f_{N+1, m+1} f_{N, m-1}\left(o_{3}+o_{1}\right)+f_{N+1, m-1} f_{N, m+1}\left(o_{1}-o_{3}\right)-2 o_{1} f_{N+1, m} f_{N, m}=0,
$$

which involves points in the $2 \times 3$ sub-lattice. (In fact this corresponds to the three-term equation (20) in [20].)

\subsubsection{Reduction of equation}

Equation (34a) is obtained from Hirota's equation (17a) by the reduction (see also [17], Part III, Example 1)

$$
\tau(n, m+1, k+1)=\tau(n, m, k),
$$

which we use to define a new function $f$ by:

$$
\tau_{n, m, k}=f_{2 n, m-k} .
$$

Defining further the new independent variables by

$$
n^{\prime}:=2 n-1, \quad m^{\prime}:=m-k,
$$

one obtains (34a) for $f$ with,

$$
o_{1}=\frac{1}{2} a(c-b), \quad o_{3}=\frac{1}{2}(a(b+c)-2 b c) .
$$

In this case the 6 corner points used in (17a) are projected to $\{(1,0),(-1,0),(-1,1),(1,-1)$, $(-1,-1),(1,1)\}$, as displayed in rightmost part of Figure 2 . 


\subsubsection{Reduction of the solution}

As for the PWF in (17b) the reduction (47) means

$$
\left(\frac{1-b q_{i}}{1-b p_{i}}\right)\left(\frac{1-c q_{i}}{1-c p_{i}}\right)=1
$$

which has the nontrivial solution

$$
q_{i}=-p_{i}+\frac{1}{b}+\frac{1}{c}
$$

After this the PWF of $(17 \mathrm{~b})$ becomes

$$
e^{\eta_{i}}=\left(\frac{a(b+c)-b c\left(a p_{i}+1\right)}{b c\left(a p_{i}-1\right)}\right)^{\left(n^{\prime}+1\right) / 2}\left(\frac{b\left(1-c p_{i}\right)}{c\left(-1+b p_{i}\right)}\right)^{m^{\prime}}
$$

We can write this in the form (45) after redefining $p \rightarrow k$ by

$$
p_{i}=\frac{1}{a} k_{i}+\frac{1}{2}\left[\frac{1}{b}+\frac{1}{c}\right]
$$

where we have used the parameterization

$$
a=c(\alpha+\beta+1), \quad b=c \frac{\alpha+\beta+1}{\alpha-\beta+1}, \quad o_{1}=-\beta b c, \quad o_{3}=\alpha b c .
$$

The phase factor $A_{i j}$ is as in (39).

\subsubsection{Special cases}

The above construction works except if $o_{1}\left(o_{1}+o_{3}\right)\left(o_{1}-o_{3}\right)=0$, corresponding to $a b c(a-$ $b)(a-c)(b-c)=0$. In these cases equation (34a) reduces to a simple $2 \mathrm{D}$ equation, which can be normalized, e.g., to $f_{N, M} f_{N+1, M+1}-f_{N+1, M} f_{N, M+1}=0$.

\subsection{Case 1b:}

We consider only Case $1 \mathrm{~b}$, i.e., $o_{4}=-\left(o_{1}+o_{2}+2 o_{3}\right)$, Case $1 \mathrm{a}$ is similar with some sign changes. Here we may assume that $o_{3}\left(o_{3}+o_{1}\right)\left(o_{3}+o_{2}\right) \neq 0$ because otherwise the system reduces to one of the 3 -term equations discussed already.

\subsubsection{Details}

The dispersion relation is given by

$$
o_{1} P_{j}^{2}+2 o_{3} P_{j} Q_{j}+o_{2} Q_{j}^{2}=o_{1}+o_{2}+2 o_{3} .
$$

In the generic case this can be resolved by

$$
P_{j}=\frac{1}{1+\alpha}\left(k_{j}+\alpha / k_{j}\right), \quad Q_{j}=\frac{1}{1+\beta}\left(k_{j}+\beta / k_{j}\right),
$$


with

$o_{1}=2 \gamma \beta(1+\alpha)^{2}, \quad o_{2}=2 \gamma \alpha(1+\beta)^{2}, \quad o_{3}=-\gamma(\alpha+\beta)(1+\alpha)(1+\beta), \quad o_{4}=2 \gamma(\alpha-\beta)^{2}$.

Here we must assume that $(1+\alpha)(1+\beta) \neq 0$. Furthemore, the three special cases $(\alpha=\beta)$, $(\alpha=0, \beta=1)$ and $(\alpha=1, \beta=0)$ may also be omitted as they lead to two-term equations.

The PWF now becomes

$$
e^{\eta_{i}}=\left[\frac{\left(k_{i}-1\right)\left(k_{i}-\alpha\right)}{\left(k_{i}+1\right)\left(k_{i}+\alpha\right)}\right]^{n}\left[\frac{\left(k_{i}-1\right)\left(k_{i}-\beta\right)}{\left(k_{i}+1\right)\left(k_{i}+\beta\right)}\right]^{m},
$$

and the phase factor

$$
\begin{aligned}
& A_{12}=\left(\frac{k_{1}-k_{2}}{k_{1}+k_{2}}\right)^{2} \times \\
& \frac{k_{1}^{2} k_{2}^{2}(\alpha+\beta-1)-\left(k_{1}^{2}+k_{2}^{2}\right) \alpha \beta+k_{1} k_{2}(\alpha+\beta)(\alpha-1)(\beta-1)+\alpha \beta(-\alpha \beta+\alpha+\beta)}{k_{1}^{2} k_{2}^{2}(\alpha+\beta-1)-\left(k_{1}^{2}+k_{2}^{2}\right) \alpha \beta-k_{1} k_{2}(\alpha+\beta)(\alpha-1)(\beta-1)+\alpha \beta(-\alpha \beta+\alpha+\beta)} .
\end{aligned}
$$

Note that $\alpha, \beta, \gamma$ as determined from (50) are not unique, but rather one can change $\alpha \mapsto 1 / \alpha, \beta \mapsto 1 / \beta, \gamma \mapsto \gamma \alpha^{2} \beta^{2}$. If this is accompanied with $k_{i} \mapsto 1 / k_{i}$ then equations (51a,51b) do not change.

\subsubsection{Reduction from Miwa's equation}

For Case 1 the equation has four terms and therefore one should be able to obtain (32b) as a reduction of Miwa's equation (18a). Let us consider the reduction

$$
\tau(n, m, k)=\tau(n+1, m+1, k+1),
$$

and define

$$
\tau_{n, m, k}=f_{n-k, k-m}
$$

and change variables by

$$
n^{\prime}=n-k, m^{\prime}=k-m,
$$

This projection takes the corners of the cube on which (18a) is defined to the points given on the left of Figure 2, in particular the diagonal $\{(0,0,0),(1,1,1)\}$ projects to $(0,0)$. Then for the equation we obtain (32b) for $n^{\prime}, m^{\prime}$ (we drop the primes in the following) with the parameter correspondence

$$
o_{1}=2 a\left(b^{2}-c^{2}\right), o_{2}=2 b\left(c^{2}-a^{2}\right), o_{3}=(a-b)(a+c)(b+c), o_{4}=-2 c\left(a^{2}-b^{2}\right) .
$$

Here we may assume that $\left(a^{2}-b^{2}\right)\left(b^{2}-c^{2}\right)\left(c^{2}-a^{2}\right) \neq 0$, because otherwise we get a two-term equation (22) with $c_{1}+c_{2}=0$.

The solution to Miwa's equation (18a) is given through $(18 \mathrm{~b}, 18 \mathrm{c})$ and the reduction (52a) implies the condition

$$
\frac{\left(1-a p_{i}\right)\left(1-a q_{i}\right)}{\left(1+a p_{i}\right)\left(1+a q_{i}\right)} \frac{\left(1-b p_{i}\right)\left(1-b q_{i}\right)}{\left(1+b p_{i}\right)\left(1+b q_{i}\right)} \frac{\left(1-c p_{i}\right)\left(1-c q_{i}\right)}{\left(1+c p_{i}\right)\left(1+c q_{i}\right)}=1
$$


and since $p_{i}+q_{i} \neq 0$ this leads to the bi-quadratic

$$
a b c(a b+a c+b c) p_{i}^{2} q_{i}^{2}+a b c\left(p_{i}^{2}+q_{i}^{2}\right)+(a+b)(b+c)(c+a) p_{i} q_{i}+a+b+c=0,
$$

which suggests an elliptic parameterization (if $a b c \neq 0$ ). This form of solution agrees with (51a), if in addition to (54) we have

$$
\begin{gathered}
\frac{\left(k_{i}-1\right)\left(k_{i}-\alpha\right)}{\left(k_{i}+1\right)\left(k_{i}+\alpha\right)}=\frac{\left(1-a p_{i}\right)\left(1-a q_{i}\right)}{\left(1+a p_{i}\right)\left(1+a q_{i}\right)}, \\
\frac{\left(k_{i}-1\right)\left(k_{i}-\beta\right)}{\left(k_{i}+1\right)\left(k_{i}+\beta\right)}=\frac{\left(1+b p_{i}\right)\left(1+b q_{i}\right)}{\left(1-b p_{i}\right)\left(1-b q_{i}\right)} .
\end{gathered}
$$

From these 3 equations one should, in principle, be able to determine $p_{i}, q_{i}$ in terms $k_{i}$ and $\alpha, \beta$ in terms of $a, b, c$, but this does not lead to simple expressions for them. In any case we have verified that the equations are consistent provided that $\alpha, \beta$ are determined from

$$
\hat{r} \alpha+\hat{s} \beta=1, \quad \hat{r} / \alpha+\hat{s} / \beta=1, \quad \text { where } \quad \hat{r}:=\frac{b(a-c)^{2}}{c(a-b)^{2}}, \quad \hat{s}:=\frac{a(b-c)^{2}}{c(b-a)^{2}} .
$$

The same equations are obtained by equating (50) and (53). Furthermore, one can show that the forms for $A_{i j}$ given in (18c) and (51b) agree, subject to relations (55) and (56).

\subsubsection{Special cases}

The discussion above holds in the generic case, where in particular the curve defined by the dispersion relation is irreducible. In fact the Jacobian of the transformation (50) is given by

$$
\left|\frac{\partial\left(o_{1}, o_{2}, o_{3}\right)}{\partial(\alpha, \beta, \gamma)}\right|=-4 \gamma^{2}(\alpha-\beta)^{2}(1+\alpha)^{2}(1+\beta)^{2}=4\left(o_{1} o_{2}-o_{3}^{2}\right) .
$$

Similarly for (53) we get

$$
\left|\frac{\partial\left(o_{1}, o_{2}, o_{3}\right)}{\partial(a, b, c)}\right|=\left(a^{2}-b^{2}\right)\left(b^{2}-c^{2}\right)\left(c^{2}-a^{2}\right)=3 o_{1} o_{2} o_{4} /(a b c) .
$$

We need to consider separately the cases where the RHS vanishes. It is perhaps not so evident that in the second case the Jacobian also vanishes when $o_{3}+o_{4}=0$, since $o_{3}+o_{4}=(a-b)(a-c)(b-c)$. Among the various special limits we are only interested in those not included in Cases 2 or 3 .

We now consider these special cases.

- Special case $o_{1} o_{2}=o_{3}^{2}, o_{i} \neq 0$ : This is a singular point for the parametrization (50) in the analysis of Sec. 4.3.1.

We use the relation to eliminate $o_{2}$. Then equation $(32 \mathrm{~b})$ becomes

$$
\begin{aligned}
f_{n-1, m+1} f_{n+1, m-1} o_{1} o_{3}-f_{n-1, m} f_{n+1, m} o_{3}\left(o_{1}+o_{3}\right)- & f_{n, m-1} f_{n, m+1} o_{1}\left(o_{1}+o_{3}\right) \\
& +f_{n, m}^{2}\left(o_{1}^{2}+o_{1} o_{3}+o_{3}^{2}\right)=0
\end{aligned}
$$


and the dispersion relation (48)

$$
\left(o_{1} P_{j}+o_{3} Q_{j}\right)^{2}=\left(o_{1}+o_{3}\right)^{2}
$$

which is solved by

$$
P_{j}=-o_{3}^{\prime} Q_{j}+\sigma_{j}\left(1+o_{3}^{\prime}\right), \quad \sigma_{j}^{2}=1, \quad o_{3}^{\prime}=o_{3} / o_{1} .
$$

Clearly there can be two kinds of solitons, depending on the chosen sign $\sigma_{j}$.

By directly solving (57) one finds the PWF

$$
e^{\eta_{j}}=\left(\frac{-o_{3}^{\prime} Q_{j}+\sigma_{j}\left(1+o_{3}^{\prime}\right)-1}{-o_{3}^{\prime} Q_{j}+\sigma_{j}\left(1+o_{3}^{\prime}\right)+1}\right)^{n}\left(\frac{Q_{j}-1}{Q_{j}+1}\right)^{m}
$$

and the phase factor

$$
A_{i j}=\frac{Q_{i}^{2}-\left(\frac{1}{2} \sigma_{i} \sigma_{j}+\frac{3}{2}\right) Q_{i} Q_{j}+Q_{j}^{2}-\left(Q_{i}-Q_{j}\right)\left(\sigma_{i}-\sigma_{j}\right)\left(\frac{1}{2}+\frac{1}{o_{3}^{\prime}}\right)-\frac{1}{2}\left(1-\sigma_{i} \sigma_{j}\right)}{Q_{i}^{2}-\left(\frac{1}{2} \sigma_{i} \sigma_{j}-\frac{3}{2}\right) Q_{i} Q_{j}+Q_{j}^{2}-\left(Q_{i}+Q_{j}\right)\left(\sigma_{i}+\sigma_{j}\right)\left(\frac{1}{2}+\frac{1}{o_{3}^{\prime}}\right)-\frac{1}{2}\left(1+\sigma_{i} \sigma_{j}\right)} .
$$

The results (58) can be obtained from (51) with the singular limit $\varepsilon \rightarrow 0$ using

$$
\begin{aligned}
\alpha & =-1+2 \varepsilon o_{1}+O\left(\varepsilon^{2}\right) \\
\beta & =-1-2 \varepsilon o_{3}+O\left(\varepsilon^{2}\right) \\
\gamma & =-1 /\left(8 \varepsilon^{2} o_{1}\right)+O\left(\varepsilon^{-1}\right)
\end{aligned}
$$

and

$$
k_{i}=\sigma_{i}-\varepsilon O_{3}\left(Q_{i}-\sigma_{i}\right)+O\left(\varepsilon^{2}\right),
$$

where $\varepsilon$ is defined by $o_{1} o_{2}-o_{3}^{2}=-\varepsilon^{2} o_{3}^{2}\left(o_{1}+o_{3}\right)^{2}$.

As for the the Miwa's parametrization (53) the relation $o_{1} o_{2}=o_{3}^{2}$ is a regular point. For convenience let us use the parametrization

$$
a=v(1+s) /(3 s-1), \quad b=v(1-s) /(3 s+1), \quad c=-s v,
$$

we get

$$
o_{1}=-2 \frac{\left(3 s^{2}+1\right)(s+1)^{2} v^{3}}{(3 s+1)^{2}}, \quad o_{2}=-2 \frac{\left(3 s^{2}+1\right)(s-1)^{2} v^{3}}{(3 s-1)^{2}}, \quad o_{3}=2 \frac{\left(3 s^{2}+1\right)\left(s^{2}-1\right) v^{3}}{9 s^{2}-1},
$$

where $v, s$ are the new parameters. Then the reduction condition (54) is satisfied by choosing

$$
p_{i}=\frac{\sigma_{i}}{v} \frac{3 \ell_{i}-1}{\ell_{i}+1}, \quad q_{i}=\frac{\sigma_{i}}{v} \frac{3 \ell_{i}+1}{\ell_{i}-1}
$$

which again shows the possibility of two different kinds of solitons, due to $\sigma_{i}= \pm 1$. 
With these choices the phase factor and the PWFs of (18) only depend on $\ell^{2}$ and it is then convenient to replace

$$
\ell_{i}^{2}=-\frac{1}{3} \frac{k_{i}^{\prime}+4 r}{k_{i}^{\prime}-4 r}, \quad r:=-\frac{2\left(3 s^{2}+1\right)^{2}(s+1) v^{3}}{(3 s+1)^{2}(3 s-1)},
$$

and with this we get form (18) the symmetric formulae (recalling (52c) and dropping the primes)

$$
\begin{gathered}
e^{\eta_{j}}=\left(\frac{k_{i}^{\prime}+\left(o_{1}+2 o_{3}\right)-3 \sigma_{j} o_{1}}{k_{i}^{\prime}+\left(o_{1}+2 o_{3}\right)+3 \sigma_{j} o_{1}}\right)^{n}\left(\frac{k_{i}^{\prime}-\left(2 o_{1}+o_{3}\right)+3 \sigma_{j} o_{3}}{k_{i}^{\prime}-\left(2 o_{1}+o_{3}\right)-3 \sigma_{j} o_{3}}\right)^{m}, \\
\left(A_{i j}\right)^{\sigma_{i} \sigma_{j}}=\frac{\left(k_{i}^{\prime}-k_{j}^{\prime}\right)^{2}}{k_{1}^{\prime 2}+k_{1}^{\prime} k_{2}^{\prime}+{k_{2}^{\prime}}^{2}-12\left(o_{1}^{2}+o_{1} o_{3}+o_{3}^{2}\right)} .
\end{gathered}
$$

We note that $r$ solves

$$
r^{2}=o_{1}^{2}+o_{1} o_{3}+o_{3}^{2}
$$

which appears later as well, and that the transformation (59) is singular if $r=0$. Finally the result (60) is related to (58) via

$$
k_{i}^{\prime}=-3 \sigma_{i} o_{3} Q_{i}+2 o_{1}+o_{3} .
$$

- Special case $o_{3}+o_{4}=0$ : (i.e., $\left.o_{1}+o_{2}+o_{3}=0\right)$. This is a regular point for the derivation of Sec. 4.3.1 but a singular point for Sec. 4.3.2. It leads to a 3-term equation,

$$
o_{3} f_{n+1, m-1} f_{n-1, m+1}+o_{1} f_{n+1, m} f_{n-1, m}-\left(o_{1}+o_{3}\right) f_{n, m+1} f_{n, m-1}=0,
$$

which is different from the 3-term equations in Cases 2 and 3.

In the analysis of Sec. 4.3.1 we should just parameterize $\alpha, \beta, \gamma$ by $\alpha=\left(o_{1}+r\right)^{2} /\left(o_{3}\left(o_{1}+o_{3}\right)\right), \beta=-\left(r+o_{1}+o_{3}\right)^{2} /\left(o_{1} o_{3}\right), \gamma=-\left(2 r^{2}-o_{3}^{2}-r\left(2 o_{1}+o_{3}\right)\right)^{2} /\left(8 o_{3} r^{2}\right)$, where $r^{2}$ is as before (61). If we now reparametrize $k_{j}=\kappa_{j}\left(2 o_{1}+o_{3}+2 r\right) /\left(3 o_{3}\right)$ then the PWFs (51a) become ${ }^{2}$

$$
\begin{aligned}
e^{\eta_{j}}= & {\left[\frac{\left(o_{3} \kappa_{j}+2 o_{1}+o_{3}-2 r\right)\left(o_{2} \kappa_{j}+2 o_{1}+o_{2}+2 r\right)}{\left(o_{3} \kappa_{j}-2 o_{1}-o_{3}+2 r\right)\left(o_{2} \kappa_{j}-2 o_{1}-o_{2}-2 r\right)}\right]^{n} } \\
& \times\left[\frac{\left(o_{3} \kappa_{j}+2 o_{1}+o_{3}-2 r\right)\left(o_{1} \kappa_{j}+2 o_{3}+o_{1}+2 r\right)}{\left(o_{3} \kappa_{j}-2 o_{1}-o_{3}+2 r\right)\left(o_{1} \kappa_{j}-2 o_{3}-o_{1}-2 r\right)}\right]^{m}
\end{aligned}
$$

and $A_{i j}(51 \mathrm{~b})$ factors as

$$
A_{i j}=\frac{\left[\left(\kappa_{i} \kappa_{j}-3\right)^{2}-\left(\kappa_{i}+\kappa_{j}\right)^{2}\right]\left(\kappa_{i}-\kappa_{j}\right)^{2}}{\left[\left(\kappa_{i} \kappa_{j}+3\right)^{2}-\left(\kappa_{i}-\kappa_{j}\right)^{2}\right]\left(\kappa_{i}+\kappa_{j}\right)^{2}} .
$$

\footnotetext{
${ }^{2}$ For these computations it is convenient to use the rational parametrization $o_{1}=z\left(9 w^{2}-1\right) /\left(3 w^{2}+\right.$ $1), o_{2}=[4(3 w-1) w z] /\left[\left(3 w^{2}+1\right)(w-1)\right], o_{3}=-\left[(3 w-1)^{2}(w+1) z\right] /\left[\left(3 w^{2}+1\right)(w-1)\right]$ and correspondingly $\alpha=(w+1) /[(3 w-1) w], \beta=[(3 w+1)(w+1)] /[(3 w-1)(w-1)], \gamma=z\left[(3 w-1)^{4}(w-1) w^{2}\right] /\left[2\left(3 w^{2}+\right.\right.$ $\left.1)^{3}(w+1)\right], r=-(3 w-1) z /(w-1)$, where $w, z$ are the two new free parameters.
} 
For the singular limit that should be used with Sec. 4.3.2 we start with

$$
o_{1}=-\left(o_{2}+o_{3}\right)\left(1+o_{2} o_{3} \epsilon^{6}\right),
$$

and get the expansions ${ }^{3}$

$$
\begin{aligned}
& a=1+\frac{2}{3}\left(o_{3}-o_{2}\right) \epsilon^{3}+O\left(\epsilon^{6}\right), \\
& b=1-\frac{2}{3}\left(o_{2}+2 o_{3}\right) \epsilon^{3}+O\left(\epsilon^{6}\right), \\
& c=1+\frac{2}{3}\left(2 o_{2}+o_{3}\right) \epsilon^{3}+O\left(\epsilon^{6}\right),
\end{aligned}
$$

and

$$
\begin{aligned}
& p(\kappa)=-\sigma-2 \frac{2 r \kappa\left(2 o_{1}+o_{3}\right)\left[3\left(\kappa^{2}-1\right) o_{3}-4 \kappa \sigma r\right]+3 o_{3} \sigma r\left(\kappa^{2}+1\right)\left[\left(\kappa^{2}-1\right) o_{3}+4 \kappa r \sigma\right]}{3\left[16 \kappa^{2} r^{2}+3 o_{3}^{2}\left(\kappa^{2}-1\right)^{2}\right]} \epsilon^{3}+O\left(\epsilon^{6}\right), \\
& q(\kappa)=-p(-\kappa) .
\end{aligned}
$$

Then in the limit $\epsilon \rightarrow 0$ one obtains (63) from (18).

- Special case $o_{1}=\omega, o_{2}=\omega^{2}, o_{3}=1, \omega^{2}+\omega+1=0 \quad$ The combination of the previous two cases implies that $o_{i}$ are cubic roots of unity. For convenience we choose $o_{j}=\omega^{j}$, where $\omega^{3}=1, \omega \neq 1$, and then the equation becomes

$$
f_{n-1, m+1} f_{n+1, m-1}+\omega f_{n-1, m} f_{n+1, m}+\omega^{2} f_{n, m-1} f_{n, m+1}=0 .
$$

For analyzing this case we have to take a singular limit in both methods. We approach the limit with $o_{1}=\omega+\epsilon^{6}, \epsilon \rightarrow 0$, and then we get the expansions $\left(\varepsilon=i \omega^{2} \epsilon\right)$

$$
\begin{aligned}
& \alpha=-1-2 i \omega \epsilon^{3}+2 \omega^{2} \epsilon^{6}+O\left(\epsilon^{9}\right), \\
& \beta=-1+2 i \epsilon^{3}+2 \epsilon^{6}+O\left(\epsilon^{9}\right), \\
& \gamma=\frac{1}{8} \omega^{2} \epsilon^{-6}-i \frac{1}{4}(\omega+2) \epsilon^{-3}+O(1)
\end{aligned}
$$

and (directly or as limits of the $o_{3}+o_{4}=0$ case)

$$
\begin{aligned}
& a=1+\frac{2}{3}(\omega+2) \epsilon^{3}-\frac{1}{6}(3 \omega+4) \epsilon^{6}+O\left(\epsilon^{9}\right), \\
& b=1+\frac{2}{3}(\omega-1) \epsilon^{3}-\frac{5}{6} \omega \epsilon^{6}+O\left(\epsilon^{9}\right), \\
& c=1-\frac{2}{3}(2 \omega+1) \epsilon^{3}-\frac{1}{6}(\omega+4) \epsilon^{6}+O\left(\epsilon^{9}\right) .
\end{aligned}
$$

Next in order to have nontriial PWFs and relations (55) we must also take singular limits in $k, p, q$, namely

$$
\begin{aligned}
& k_{j}=\sigma_{j}\left[1+i \frac{1}{3} \epsilon^{3}\left(-k_{j}^{\prime}+2(\omega-1)\right)\right], \\
& p_{j}=\sigma^{\prime}+2 \frac{\epsilon^{3}}{k_{j}^{\prime}}\left(\sigma_{j}(1-\omega)+\sigma^{\prime}(\omega+1)\right)+O\left(\epsilon^{6}\right), \\
& q_{j}=-\sigma^{\prime}+2 \frac{\epsilon^{3}}{k_{j}^{\prime}}\left(\sigma_{j}(1-\omega)-\sigma^{\prime}(\omega+1)\right)+O\left(\epsilon^{6}\right) .
\end{aligned}
$$

\footnotetext{
${ }^{3}$ This is also the type of limit by which Miwa's equation reduces to the Hirota's equations.
} 
Here $\sigma_{j}, \sigma^{\prime}= \pm 1$. In the limit $\epsilon \rightarrow 0$ we then get from (18)

$$
e^{\eta_{j}}=\left(\frac{k_{j}^{\prime} \omega^{2}-2 \omega-1-3 \sigma_{j}}{k_{j}^{\prime} \omega^{2}-2 \omega-1+3 \sigma_{j}}\right)^{n}\left(\frac{k_{j}^{\prime}-2 \omega-1+3 \sigma_{j}}{k_{j}^{\prime}-2 \omega-1-3 \sigma_{j}}\right)^{m},
$$

and

$$
\left(A_{i j}\right)^{\sigma_{i} \sigma_{j}}=\frac{\left(k_{i}^{\prime}-k_{j}^{\prime}\right)^{2}}{{k_{i}^{\prime}}^{2}+k_{i}^{\prime} k^{\prime}{ }_{j}+{k_{j}^{\prime}}^{2}},
$$

which also follow directly form (60). These also follow as singular limits from (63), if we use $\kappa_{j}=-\sigma_{j}(2 \omega+1)\left(1+i \epsilon^{3} k_{j}^{\prime} / 3\right)$, along with $o_{1}=\omega+\epsilon^{6}, o_{2}=\omega^{2}-\frac{4}{7}(2 \omega+3) \epsilon^{6}, o_{3}=$ $-o_{1}-o_{2}+O\left(\epsilon^{6}\right)$.

- Special case $o_{1}=0, o_{2} o_{4} \neq 0$ : This can be obtained from the analysis of 4.3 .1 by just putting $\beta=0$ but for (53) a singular limit is needed. Thus we let

$$
\begin{gathered}
\alpha=o_{4} / o_{2}, \beta=\epsilon^{3} o_{4} /\left(4 o_{3}^{2}\right), \gamma=o_{2}^{2} /\left(2 o_{4}\right), \\
a=a_{m} / \epsilon, b=-\epsilon^{2} o_{2} /\left(2 a_{m}^{2}\right), c=-\epsilon^{2} o_{4} /\left(2 a_{m}^{2}\right), \text { where } a_{m}^{3}=-2 o_{3}\left(o_{2}+o_{3}\right) .
\end{gathered}
$$

Then with the parametrization

$$
p_{i}=\frac{-4\left(o_{2}+o_{3}\right) o_{3}}{a_{m} O_{2} \epsilon^{2} k_{i}}, q_{i}=\frac{-\left(o_{4}+o_{2} k_{i}^{2}\right) \epsilon}{2 a_{m} o_{3} k_{i}},
$$

we find that in the limit $\epsilon \rightarrow 0$ equation (53) is satisfied and the formulae of Sec. 4.3.2 work. From both approaches the result is

$$
e^{\eta_{i}}=\left(\frac{\left(k_{i}-1\right)\left(k_{i}-\alpha\right)}{\left(k_{i}+1\right)\left(k_{i}+\alpha\right)}\right)^{n}\left(\frac{k_{i}-1}{k_{i}+1}\right)^{m}, \quad A_{12}=\frac{\left(k_{1}-k_{2}\right)^{2}\left(k_{1} k_{2}-\alpha\right)}{\left(k_{1}+k_{2}\right)^{2}\left(k_{1} k_{2}+\alpha\right)} .
$$

The difference equation in this sub-case reads

$$
\left(f_{n+1, m-1} f_{n-1, m+1}-f_{n, m+1} f_{n, m-1}\right) o_{3}-\left(f_{n+1, m} f_{n-1, m}-f_{n, m}^{2}\right)\left(o_{3}+o_{2}\right)=0 .
$$

The case $o_{1}=0, o_{2} o_{4} \neq 0$ is similar.

The further specialization to $o_{1}=o_{4}=0$ can be obtained from the above by letting first $o_{2}=-2 o_{3}+\rho \epsilon$, i.e., $o_{4}=-\rho \epsilon-\epsilon^{3}$. This corresponds to $\alpha \rightarrow 0$ in (65) and

$$
f_{n+1, m-1} f_{n-1, m+1}-f_{n, m+1} f_{n, m-1}+f_{n+1, m} f_{n-1, m}-f_{n, m}^{2}=0 .
$$

Similarly the case $o_{1}=o_{2}=0$ can be obtained by further putting $o_{2}=\epsilon$. This corresponds to $\alpha \rightarrow \infty$, which yields

$$
e^{\eta_{i}}=\left(\frac{1-k_{i}}{1+k_{i}}\right)^{n}\left(\frac{k_{i}-1}{k_{i}+1}\right)^{m}, \quad A_{12}=-\left(\frac{k_{1}-k_{2}}{k_{1}+k_{2}}\right)^{2},
$$

and

$$
f_{n+1, m-1} f_{n-1, m+1}-f_{n, m+1} f_{n, m-1}-f_{n+1, m} f_{n-1, m}+f_{n, m}^{2}=0 .
$$

Note that here $A_{12}$ is negative so this system can have a non-singular 2SS only if it composed from a complex conjugate pair of parameters $k_{i}$. In both of these double zero cases the solitons have a fixed angle in the $n, m$-space. 
- Special case $o_{4}=0\left(o_{1}+o_{2}+2 o_{3}=0\right)$ : This is a regular point for Sec. 4.3.2 but a singular point for Sec. 4.3.1. The dispersion relation is

$$
(P-Q)\left(o_{1} P-o_{2} Q\right)=0
$$

and thus we have two kinds of solitons if $o_{2} \neq o_{1}$. The nontrivial factor $\left(o_{1} P-o_{2} Q\right)$ is included in the parametrization (49) provided that $k_{i} \rightarrow 0$ together with $o_{4} \rightarrow 0$. We will therefore take $\epsilon:=o_{4}, k_{i}=k_{i}^{\prime} \epsilon /\left(o_{1}-o_{2}\right)^{2}$ with $k_{i}^{\prime}$ arbitrary (the $\left(o_{1}-o_{2}\right)^{2}$ factor is for simplicity later $)$. For the remaining parameters we construct a power series expansion in $\epsilon$ :

$$
\alpha=\frac{o_{2}}{\left(o_{1}-o_{2}\right)^{2}} \epsilon+\ldots, \quad \beta=\frac{o_{1}}{\left(o_{1}-o_{2}\right)^{2}} \epsilon+\ldots, \quad \gamma=\frac{1}{2}\left(o_{1}-o_{2}\right)^{2} / \epsilon+\ldots
$$

The limit of the PWF is given by

$$
e^{\eta_{i}}=\left(\frac{o_{2}-k_{i}^{\prime}}{o_{2}+k_{i}^{\prime}}\right)^{n}\left(\frac{o_{1}-k_{i}^{\prime}}{o_{1}+k_{i}^{\prime}}\right)^{m}
$$

the phase factor $A_{i j}$ gets the form

$$
A_{i j}=\left(\frac{k_{i}^{\prime}-k_{j}^{\prime}}{k_{i}^{\prime}+k_{j}^{\prime}}\right)^{2}\left(\frac{o_{2} o_{1}+k_{1}^{\prime} k_{2}^{\prime}}{o_{2} o_{1}-k_{1}^{\prime} k_{2}^{\prime}}\right)
$$

and the bilinear equation $(32 \mathrm{~b})$ reads

$$
\left(o_{2}+o_{1}\right) f_{n+1, m-1} f_{n-1, m+1}+\left(o_{2}-o_{1}\right) f_{n+1, m} f_{n-1, m}-\left(o_{2}-o_{1}\right) f_{n, m+1} f_{n, m-1}-\left(o_{2}+o_{1}\right) f_{n, m}^{2}=0 \text {. }
$$

In order to keep the connection to the Miwa equation as given in Equations (53-56) we need first of all the expansions for $a, b, c$. The leading terms are

$$
a=a_{0}+\ldots, \quad b=-a_{0} \frac{o_{1}}{o_{2}}+\ldots, \quad c=a_{0} \frac{o_{1}}{o_{1}^{2}-o_{2}^{2}} \epsilon+\ldots, \text { where } a_{0}^{3}=\frac{o_{2}^{2}}{2 o_{1}} .
$$

Finally to connect $p_{i}, q_{i}$ in (54-55b) to $k_{i}^{\prime}$ we find for $p_{i}, q_{i}$ the expansions

$$
p_{i}=\frac{o_{2}}{a_{0} k_{i}^{\prime}}+\ldots, \quad q_{i}=\frac{2}{\epsilon} \frac{\left(o_{1}+o_{2}\right)\left(o_{1}-o_{2}\right)^{2} a_{0}^{2} k_{i}^{\prime}}{\left(k_{i}^{\prime 2}-o_{1} o_{2}\right) o_{2}^{2}}+\ldots
$$

It is easy to see that this satisfies (55) but in order to satisfy (54) is actually necessary to compute the second order in the $\epsilon$-expansions, while for (56) three orders are necessary.

- Special case $o_{4}=0$ with others. The special case where $o_{4}=0$ together with $o_{2}=o_{1}$, or $o_{1}=0$, or $o_{2}=0$ leads to dispersion relations $Q(P-Q)=0$ and $P(P-Q)=0$, respectively, which are perhaps too simple to be of interest. 


\section{Conclusions}

Hirota's direct method has turned out to be very efficient in deriving soliton solutions for a given equation. It has also be used as a method for searching for integrable equations in the continuous case, the criterion being the existence of three-soliton solutions.

Here we have applied the three-soliton condition to two-dimensional partial difference equations. In comparison with the continuous case there is now much more freedom, since the same partial derivative can be discretized in many ways. In this paper we restricted the search to partial-difference equations defined on a $3 \times 3$ stencil. For the $2 \times N$ case, see $[20]$.

The master bilinear P $\Delta$ Es are Hirota's three-term DAGTE equation (17a) and Miwa's four term equation (18a). In the detailed analysis of the results found here we showed that all of them can be obtained from either one of these master equations with a suitable projection. However, sometimes a nontrivial singular parameter limit was also needed. Since the phase factor of the soliton solution is the same for a discrete equation and its continuum limit we can say that most of the results can be considered as discrete versions

of the KdV equations, but some are related to the Boussinesq or Ito equations, while some cannot yet be definitely identified.

\section{Acknowledgments}

This project is partially supported by the NSF of China (No. 11071157), SRF of the DPHE of China (No. 20113108110002) and Shanghai Leading Academic Discipline Project (No. J50101).

\section{References}

[1] M.J. Ablowitz and P.A. Clarkson, Solitons, Nonlinear Evolution Equations and Inverse Scattering, (Cambridge Univ. Press, 1991). Integrability, ed. A. Mikhailov, Lecture Notes in Physics 767, (Springer, 2009)

[2] J. Nonlin. Math. Phys. 10, Suppl. 2, eds. F.W. Nijhoff, Yu. Suris and C.-M. Viallet (2003); J. Nonlin. Math. Phys. 12, Suppl. 2, eds. J.F. van Diejen and R. Halburd (2005); J. Phys. A: Math. Theor. 40 Nr. 42, eds. A. Doliwa, R. Korhonen and S. Lafortune. (2007); J. Phys. A: Math. Theor. 42, Nr. 45, eds. D. Levi, P. Olver, Z. Thomova and P. Winternitz (2009).

[3] B. Grammaticos, A. Ramani and V.G. Papageorgiou, Do integrable mappings have the Painlevé property?, Phys. Rev. Lett. 67 1825-1828 (1991). A. Ramani, B. Grammaticos and J. Hietarinta: Discrete versions of the Painlevé Equation, Phys. Rev. Lett. 67, 1829-1831 (1991).

[4] J.C. Nimmo and W.K. Schief, Superposition principles associated with the Moutard transformation: an integrable discretization of a (2+1)-dimensional sine-Gordon sys- 
tem, Proc. R. Soc. Lond. A 453 (1997) 255-279; F.W. Nijhoff, A. Ramani, B. Grammaticos and Y. Ohta, On discrete Painlevé equations associated with the lattice KdV systems and the Painlevé VI equation, Stud. Appl. Math. 106 261-314 (2001); F.W. Nijhoff and A.J. Walker, The discrete and continuous Painlevé VI hierarchy and the Garnier systems, Glasgow Math. J. 43A (2001) 109-123; F.W. Nijhoff, Lax pair for the Adler (lattice Krichever-Novikov) system, Phys. Lett. 297A (2002), 49-58; V.E. Adler, A.I. Bobenko and Yu.B. Suris, Classification of integrable equations on quadgraphs, the consistency approach, Commun. Math. Phys. 233 (2003) 513-543.

[5] G. Falqui and C.-M. Viallet, Singularity, Complexity, and Quasi-Integrability of Rational Maps, Commun. Math. Phys. 154, 111-125 (1993); J. Hietarinta and C.-M. Viallet: Singularity confinement and chaos in discrete systems, Phys. Rev. Lett. 81, 325-328 (1998). Y.Ohta, K.M. Tamizhmani, B.Grammaticos and A.Ramani: Singularity confinement and algebraic entropy: the case of the discrete Painleve equations, Phys. Lett. A 262, 152-157 (1999). J. Hietarinta and C.-M. Viallet: Discrete Painlevé $I$ and singularity confinement in projective space, Chaos, Solitons and Fractals 11, 29-32 (2000). M. Bellon and C.-M. Viallet, Algebraic Entropy, Comm. Math. Phys. 204, 425-437 (1999).

[6] R. Hirota, Discrete analogue of generalized Toda equation, J. Phys. Soc. Japan 50, 3785-3791 (1981) .

[7] T. Miwa, On Hirota's difference equations, Proc. Japan Acad. 58, 9-12 (1982).

[8] R. Hirota, Exact Solution of the Korteweg-de Vries Equation for Multiple Collision of Solitons, Phys. Rev. Lett 27, 1192-1194 (1971).

[9] R. Hirota, The Direct Method in Soliton Theory, Cambridge Tracts in Mathematics 155, (Cambridge Univ. Press, 2004).

[10] R. Hirota and J. Satsuma, A variety of nonlinear network equations generated from the Bäcklund transformation for the Toda lattice, Prog. Theor. Phys., Suppl. 59, 64100 (1976).

[11] B. Grammaticos, A. Ramani and J.Hietarinta, Multilinear operators: the natural extension of Hirota's bilinear formalism, Phys. Lett. A 190, 65-70 (1994).

[12] C. Gilson, J. Hietarinta, J. Nimmo and Y. Ohta: Sasa-Satsuma higher-order nonlinear Schrödinger equation and its bilinearization and multisoliton solutions, Phys. Rev. E 68, 016614 (2003).

[13] A.C. Hearn, "REDUCE User's Manual", Version 3.8 (2004); http://reduce-algebra. sourceforge.net/.

[14] J. Hietarinta, A search of bilinear equations passing Hirota's three-soliton condition: I-IV, J. Math. Phys. 28, 1732-1742, 2094-2101, 2586-2592 (1987); ibid 29, 628-635 (1988). 
[15] J. Hietarinta, Recent results from the search for bilinear equations having three-soliton solutions in "Nonlinear Evolution Equations: Integrability and Spectral Methods", A. Degasperis, A.P. Fordy and M. Lakshmanan (eds.), (Manchester UP, 1990), pp. 307317.

[16] R. Hirota, Nonlinear partial difference equations I-III, J. Phys. Soc. Japan 43, 14241433, 2074-2089 (1977).

[17] E. Date, M. Jimbo and T. Miwa, Method for generating discrete soliton equations I$V$, J. Phys. Soc. Japan 51, 4116-4124, 4125-4131 (1982), 52, 388-393, 761-765,766-771 (1983).

[18] J. Atkinson, J. Hietarinta and F. Nijhoff, Soliton solutions for Q3, J. Phys. A: Math. Theor. 41, 142001 (2008).

[19] J. Hietarinta and D.J. Zhang, Soliton solutions for ABS lattice equations: II. Casoratians and bilinearization, J. Phys. A: Math. Theor. 42, 404006 (30pp.) (2009).

[20] J. Hietarinta and D.J. Zhang, Hirota's method and the search for integrable partial difference equations. 2. Equations on a $2 \times N$ stencil, Reports of RIAM Symposium No.22AO-S8, pp. 30-36 (2011). 